\title{
Reactive Oxygen Species Limit the Ability of Bone Marrow Stromal Cells to Support Hematopoietic Reconstitution in Aging Mice
}

\author{
Rahul Khatri, ${ }^{\star}$ Shyam Krishnan, ${ }^{*}$ Sushmita Roy, Saborni Chattopadhyay, \\ Vikash Kumar, and Asok Mukhopadhyay
}

Aging of organ and abnormal tissue regeneration are recurrent problems in physiological and pathophysiological conditions. This is most crucial in case of high-turnover tissues, like bone marrow (BM). Using reciprocal transplantation experiments in mouse, we have shown that self-renewal potential of hematopoietic stem and progenitor cells (HSPCs) and BM cellularity are markedly influenced with the age of the recipient mice rather than donor mice. Moreover, accumulation of excessive reactive oxygen species (ROS) in BM stromal cells compared to HSPC compartment, in time-dependent manner, suggests that oxidative stress is involved in suppression of BM cellularity by affecting microenvironment in aged mice. Treatment of these mice with a polyphenolic antioxidant curcumin is found to partially quench ROS, thereby rescues stromal cells from oxidative stress-dependent cellular injury. This rejuvenation of stromal cells significantly improves hematopoietic reconstitution in 18-month-old mice compared to age control mice. In conclusion, this study implicates the role of ROS in perturbation of stromal cell function upon aging, which in turn affects BM's reconstitution ability in aged mice. Thus, a rejuvenation therapy using curcumin, before HSPC transplantation, is found to be an efficient strategy for successful marrow reconstitution in older mice.

\section{Introduction}

$\mathrm{H}$ EMATOPOIETIC STEM CELLS (HSCs) possess extensive self-renewal and differentiation ability for lifelong support of mature blood cells. These unique properties of HSCs are maintained and regulated in the osteoblastic and endothelial niches of bone marrow (BM) in which different stromal cells play important roles [1]. Earlier, it was assumed that the self-renewal properties and the functions of HSCs did not compromise with age. Many investigators have disagreed with this aging phenomenon and concluded that the functional properties are perpetual in nature. However, subsequent studies have confirmed that aged HSCs are compromised with respect to BM homing ability [2,3], lineage commitment [4,5], self-renewal capacity [6], and further they accumulate DNA damage like other normal cells $[7,8]$. This complex process of gradual deterioration of physiological functions upon aging is virtually involved in all cells and tissues leading to morbidity and mortality of the organism. In this regard, replicative senescence, a state of irreversible growth arrest, has been proposed by many investigators, which capacitates stem cell functions [9-11]. Gradual declining of BM reconstitution ability of serially transplanted HSCs in lethally ir- radiated mice supported the above phenomenon of finite potential for self-renewal and differentiation under regenerative stress [12,13].

The question of HSC aging is further complicated by the presence of many subsets of cells within HSC compartment, and each functional property is assumed to be attributed to a specific subset of cells $[14,15]$. Furthermore, it is likely that the dominance of each subset of cells is an age-dependent phenomenon. This may explain why in old mice the balanced differentiation potential of HSCs is skewed toward myeloid lineage $[2-4,8,15]$.

Interestingly, although HSCs exhibited lower repopulation ability in the old mice, their absolute number did not change compared to young counterparts, rather they increased [4]. Later, it has been proved that increasing selfrenewal and diminishing differentiation potential of aging HSCs are epigenetically controlled [16]. Aging of hematopoietic system is manifested with increasing incidence of blood disorders, including autoimmune diseases. The emergence of age-related HSC phenotype has been explained by intrinsic factors, like DNA damage and epigenetic dysregulation [17], which cannot be reversed by therapeutic intervention. In recent past, this paradigm has shifted as the

Stem Cell Biology Laboratory, National Institute of Immunology, New Delhi, India.

*These authors contributed equally to this work.

(C) Rahul Khatri et al., 2016; Published by Mary Ann Liebert, Inc. This Open Access article is distributed under the terms of the Creative Commons Attribution Noncommercial License (http://creativecommons.org/licenses/by-nc/4.0/) which permits any noncommercial use, distribution, and reproduction in any medium, provided the original author(s) and the source are credited. 
impaired contribution of old HSC transplantation was found to partially improve by either antioxidative therapy or rapamycin treatment $[18,19]$.

Adult tissue-specific stem and progenitor cells (SPCs) are housed within the respective niches that control their selfrenewal and lineage commitment [20]; it is likely that perturbation of niche or stromal cells may lead to malfunctions in SPCs. Normal BM hematopoiesis requires a complex interplay between the HSCs and the marrow microenvironment, which is necessary for switching on/off of several proliferation and differentiation signaling cascades. Unfavorable stromal microenvironment has been implicated in many hematological diseases, like aplastic anemia [21], multiple myeloma [22], acute myeloid leukemia [23], etc. Considering this, we hypothesize that with age, BM stromal cells do not extend ample support to HSCs like in young subjects, leading to improper reconstitution after myeloablation and imbalance in lineage commitment, etc. Therefore, the aim of this study was to decipher the cause of functional alternation in hematopoietic stem and progenitor cells (HSPCs) in old mice and the role of stromal cells, if any, for such changes. In this study, we report that aging causes accumulation of reactive oxygen species (ROS) in BM stromal cells and their apoptotic death. Inadequate functioning of a part of stem cell niche thereby reduces the hematopoietic reconstitution in the aging mice.

\section{Materials and Methods}

\section{Mouse strains}

C57B1/6J [Ptprc ${ }^{b}$ (Ly5.2)], C57B1/6J SJL [Ptprc ${ }^{c}$ (Ly5.1)] and eGFP-expressing C57B1/6J syngeneic mouse strains of three age groups ( 2 months- young mice, $12 \pm 1$ month, and $18 \pm 1$-month-old mice) were used in the study. Mice were obtained from The Jackson Laboratories and maintained in individually ventilated cages, fed with autoclaved acidified water, and irradiated food ad libitum in the experimental animal facility of the institute. All experiments were conducted as per procedures approved by the Institutional Animal Ethics Committee of National Institute of Immunology, New Delhi.

\section{Transplantation of cells}

For reciprocal hematopoietic reconstitution assay, CD45.2/ CD45.1 C57B1/6J recipient mice were sublethally irradiated at $700 \mathrm{cGy}$ in two splits of $3 \mathrm{~h}$ interval. Different groups of young and old mice were individually transplanted with single dose of CD45.1/CD45.2 BM mononuclear cells (MNCs) (0.5, 1.5 , and $4.5 \times 10^{6}$ ) or $3 \times 10^{4} \mathrm{CD} 45.1 / \mathrm{CD} 45.2 \mathrm{Lin}^{-} \mathrm{Sca}-1^{+} \mathrm{c}-\mathrm{kit}^{+}$ (LSK) cells. The isolation of BM cells and purification of LSK cells were described in our previous publication [24]. Transplantation was conducted in such a way that old $(\mathrm{O})$ recipients received young $(\mathrm{Y})$ cells and vis-à-vis (scheme in Fig. 3A). The recipient mice were sacrificed after 2 months of transplantation and BM was analyzed for donor-derived HSPCs and total MNCs.

Furthermore, 24 CD45.2 old mice were equally divided into four groups: Group I \& II received vehicle and Group III \& IV were treated with curcumin $(100 \mathrm{mg} / \mathrm{kg}$ body weight dissolved in $10 \%$ DMSO-normal saline) through intraperitoneal route at every 2-day intervals for a period of
20 days. Two days after the last dose, mice belonging to Group I \& III were sacrificed to determine ROS and gene expressions in stromal cells. Since we isolated stromal cells by negative sorting technique using MACS on the basis of CD $45^{-}$phenotype, the stromal population was a heterogeneous mixture of fibroblasts, osteoblasts, and endothelial cells. We ensured complete removal of erythrocytes by treatment of cells with a lysis buffer. Group II \& IV mice were irradiated as before and each one of them was transplanted with 30,000 LSK cells (scheme in Fig. 6). After 15 days of transplantation, mice were sacrificed for analysis of total BM-MNCs, gene expression, $\mathrm{CD} 45^{-}$cells number, and ROS levels.

\section{Estimation of mitochondrial ROS and cellular apoptosis}

After staining BM-MNCs with CD45 antibody or LSK antibodies, they were resuspended in $1 \mathrm{~mL}$ of DMEM (w/o FBS, phenol red, and antibiotics). To that, $1 \mu \mathrm{L}$ MitoSOX (Sigma) reagent $(5 \mathrm{mM}$ stock solution) was added and cells were incubated at $37^{\circ} \mathrm{C}$ for $15 \mathrm{~min}$ in dark. Cells were washed in PBS and analyzed by BDFACS Aria III (BD Biosciences). To determine apoptosis, CD45 ${ }^{-}$or LSK antibody-labeled cells were stained with Annexin V antibody (BD Biosciences) before flow cytometric analysis.

\section{Estimation of GSH/GSSG level in stromal cells}

$\mathrm{CD}^{4} 5^{-}$stromal cells were isolated from curcuminuntreated and -treated old mice. Glutathione (GSH) and oxidized glutathione (GSSG) were quantified using a commercially available kit (EnzyChrom GSH/GSSG assay kit; Bio Assay Systems). Each analysis was based on $2 \times 10^{6}$ sorted cells. Cellular GSSG, GSH, and total GSH levels were calculated using specific formulas provided with the protocol.

\section{In vitro culture of stromal cells with $\mathrm{N}$-acetyle cysteine}

Two- and 18-month-old mice were sacrificed and BM stromal cells were isolated and cultured in 10\% FBSsupplemented IMDM. Thirty thousand stromal cells of young and old mice in passage 2 and passage 4, respectively, were grown overnight in 12-well plates and then treated with $\mathrm{N}$ acetyle cysteine (NAC) $(10 \mathrm{mM})$ for $48 \mathrm{~h}$. Control wells received the medium alone. After treatment, the stromal cells were washed and cocultured with $12,000 \mathrm{LSK}$ cells/well in the presence of IMDM supplemented with 5\% ES certified FBS (Biological Industries). After $72 \mathrm{~h}$ of coculture, cells were analyzed in terms of LSKFlk2 ${ }^{+/-}$, and CD29, CD44, and CXCR4 in the background of LSK cells by flow cytometry. In parallel, NAC-treated and -untreated stromal cells of young and old mice were stained with Mitosox and Annexin-V antibody to measure the effect of NAC on ROS level and apoptosis.

\section{Supplementary methods}

Methods for isolation and purification of progenitor cells, colony-forming unit (CFU) assay, GSH/GSSG estimation, flow cytometry with antibodies, and gene expression analysis with primer design are included in Supplementary Data section. 


\section{Statistics}

Results of multiple experiments are reported as mean \pm SEM (Standard Error Mean). Student's $t$-test was carried out to calculate the significance between the means of different groups and $P<0.05$ was considered significant. All analyses were carried out using GraphPad Prism software, Version 5.02.

\section{Results}

\section{HSPCs do not change with age, whereas myeloid} colony-forming potential gets increased in old mice

We analyzed HSPCs based on LSKFlk2 $2^{+-}$phenotypes [25]. LSKFlk2 ${ }^{-}$cells were found to have sustained multilineage engraftment potential, whereas, LSKFlk2 ${ }^{+}$cells support short-term multilineage engraftment [25]. Initially, we compared these two populations of HSPCs in BM of young ( 2 months) and old (18 months) mice. Although there was a minor imbalance in the LSKFlk2 ${ }^{+}$to LSKFlk2 ${ }^{-}$ratio, no significant change in these two distinct categories of cells was observed in young and old mice, suggesting that HSPC compartment remains unaffected with age in normal physiological state (Fig. 1A). Further analyses of peripheral blood MNCs revealed that old mice were myeloid biased, as combined Mac1+Gr1 populations significantly $(P<0.05)$ increased from $24 \%$ to $40 \%$. In these mice, $\mathrm{T}$ lymphoid declined, whereas B lymphoid remained unaltered in two extreme age groups of mice (Fig. 1B).

To compare the effect of aging in progenitor cells with respect to different myeloid lineages, we performed the CFU assay of BM-MNCs. As BM is a hypoxic tissue, the colony assays were performed in both normoxic $(21 \%$ ox- ygen) and hypoxic (5\% oxygen) conditions. Interestingly, in both oxygen levels, CFU granulocyte, erythrocyte, monocyte, megakaryocyte (GEMM) were found to be significantly high in old mice compared to that in young, whereas CFU-GM was more only in the normoxic culture (Fig. 1C \& Supplementary Fig. S1). Large number of CFU-E (88.8 $\pm 6.7 /$ $10^{5}$ cells) in old mice as opposed to that in young mice $\left(31.7 \pm 3.4 / 10^{5}\right.$ cells) was only observed in the hypoxic culture; this confirmed that cellular commitment to erythroid cells was more in the former mice group (Supplementary Fig. S1). Overall, the above analysis shows that HSPCs in these two extreme age groups of mice do not change, although functional progenitors get skewed toward myeloid lineage.

\section{HSPCs in old mice are exhausted rapidly than younger counterpart}

To understand whether HSPCs of young and old mice exhaust at equal rate due to demand of continuous turnover and differentiation, we conducted serial transplantation experiments using young recipients. The scheme of the experiment has been explained in Fig. 2A, where equal numbers of sorted donor LSK cells from young or old mice were transplanted in each stage and subsequently BM cellularity and total donor-derived HSPCs were determined after every 2-month intervals. It may be noted that BM cellularity of LSKFlk2 ${ }^{+/-}$phenotypes were higher in case of young donor cells compared to old donor cells in all stages of transplantation. Irrespective of the donor age, the BM cellularity was comparable in primary $\left(1^{\circ}\right)$ and secondary $\left(2^{\circ}\right)$ transplantation, which, however, significantly declined in the third $\left(3^{\circ}\right)$ stage (Fig. 2B, left bar diagrams). As the
FIG. 1. Comparative study of HSPCs and CFU-C in young and old mice. Two- and 18-month-old mice were sacrificed; BM cells were analyzed for LSKFlk2 ${ }^{+}$and LSKFlk2 ${ }^{-}$and colony-forming potential. (A) Representative dotplots show gating strategy for analysis of $\mathrm{LSKFlk} 2^{+/-}$cells, the comparative results are shown in the bar diagram $(n=6$ for each group). (B) Bar diagram shows lineage commitment of myeloid and lymphoid cells in peripheral blood samples $(n=6$ for each group). (C) Bar diagram shows myeloid colony-forming progenitors present in BM-MNCs $(n=4$ for each group). $* P<0.05$. BM, bone marrow; CFU, colony-forming unit; HSPC, hematopoietic stem and progenitor cell; MNC, mononuclear cell.
A
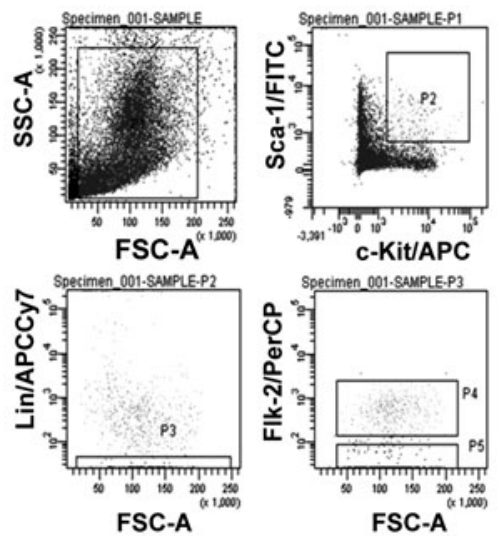

B

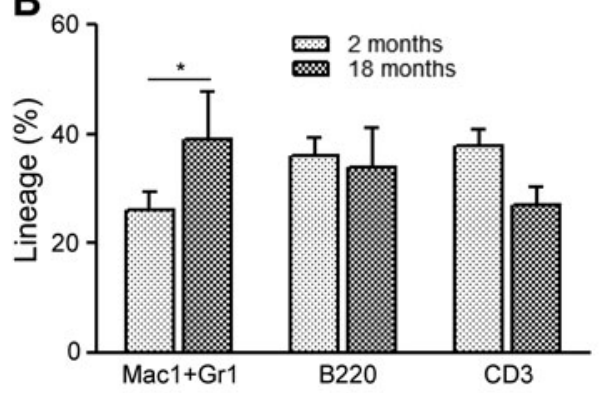

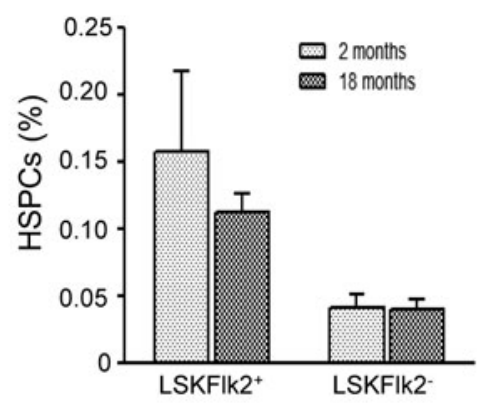

C

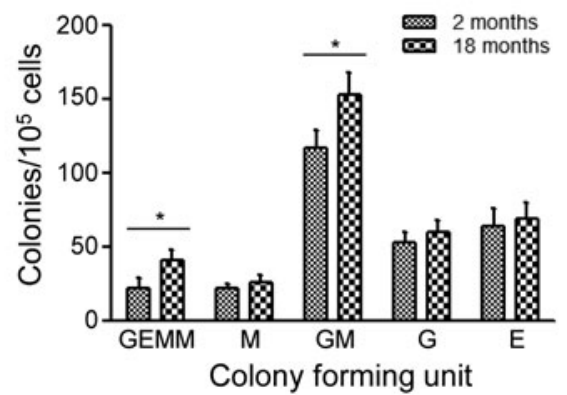



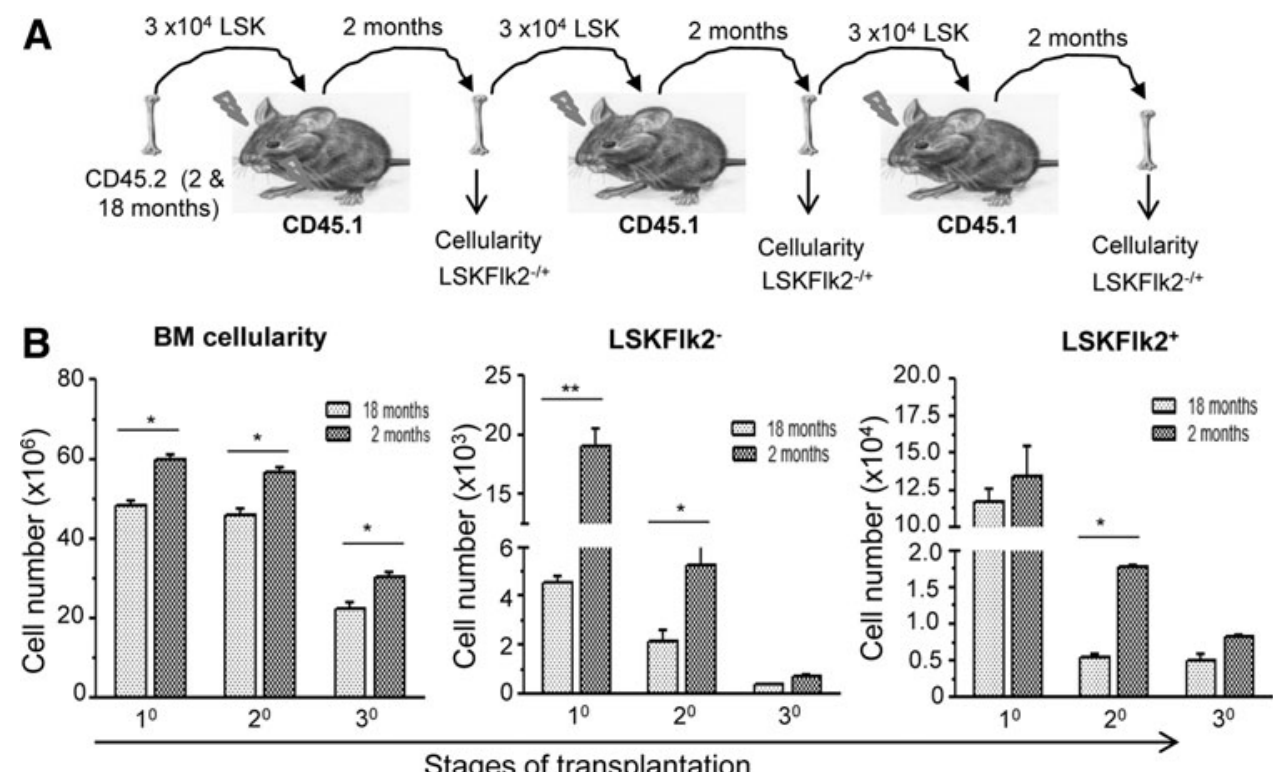

FIG. 2. Study of replicative senescence in LSK cells. (A) Scheme of the experiment shows that 30,000 CD45.2LSK cells from young and old mice were transplanted in irradiated CD45.1 young mice $\left(1^{\circ}\right)$. Two months after transplantation, each group of mice were sacrificed, and BM cellularity and LSKFlk $2^{+/-}$cells were determined from 2 tibias and 2 femurs. In the second stage $\left(2^{\circ}\right)$, again 30,000 sorted CD45.1LSK cells from each group $\left(1^{\circ}\right)$ were transplanted in new recipient mice. Mice were sacrificed, analyzed as before, and used similarly for $3^{\circ}$ transplantation. (B) Comparative total BM cellularity (left panel), LSKFlk2 ${ }^{-}$cells (middle panel), and LSKFlk $2^{+}$cells (right panel) at different stages are shown. Number of mice in each group $(n)=6 . * P<0.05 ; * * P<0.01$.

LSKFlk2 $2^{-}$population was significantly reduced from $2^{\circ}$ transplantation in both age groups of mice, we concluded that donor HSPC self-renewal potential was compromised from the first stage, resulting in an overall drop in BM cellularity after $3^{\circ}$ transplantation. The comparative study also revealed that LSKFlk2 $2^{-}$pool in both age groups of mice steadily declined, but older mice were severely affected beginning from the $1^{\mathrm{o}}$ transplantation (Fig. 2B, middle). LSKFlk $2^{+}$cells were comparable in both age groups after $1^{\mathrm{o}}$ transplantation, later both were affected, and the decrease was more pronounced in old donor cells (Fig. 2B, right). Overall, the serial transplantation experiments suggested that irrespective of the age of donor cells, both LSKFlk2 $2^{+/-}$ cell populations and BM cellularity were significantly affected. The old mice were severely affected, probably due to sustained replicative stress on HSPCs before and after first stage of transplantation.

\section{Hematopoietic reconstitution ability decreased with the age of the recipient mice}

Earlier results showed that the HSPC compartment gets exhausted with time, more prominently in case of older donor cells. However, these results did not address the potential impact of an age-dependent marrow environment on hematopoietic reconstitution. To address this, we have followed a reciprocal transplantation scheme in which different doses of BM-MNCs of young (Y) mice were transplanted in an18-month-old (O) recipient and vice versa. The results showed significantly $(P<0.01)$ low recovery of young donor-derived LSKFlk2 $2^{+/-}$cells from an old recipient (YO) than old donor-derived LSKFlk2 ${ }^{+/-}$cells recovered from a young recipient $(\mathrm{OY})$. The representative dot plots for determining LSKFlk2 $2^{+-}$cells are shown in Supplementary
Fig. S2A, B. These declining trends were prominent in the case of $\mathrm{LSKFlk}^{+}$cells in all transplantation doses (Fig. 3A). If the age of the donor stem cells solely dictates marrow reconstitution, the older recipients are supposed to show greater HSPCs with normal BM cellularity, as they received young donor cells. Intriguingly, we observed opposite results, suggesting that perhaps age of the donor cells alone is not crucial in this case; the BM microenvironment indeed plays an important role. We have not seen proper dose response with the recovery of LSKFlk $2^{+/-}$cells; this possibly was due to large number of cells transplanted and duration of the experiments was relatively longer ( 2 months).

Since above experiments were conducted using BMMNCs, which contain subsets of stromal cells, it is possible that there might be an effect of donor-derived stromal cells on overall outcome of the reciprocal transplantation experiments. To avoid this, we repeated the reciprocal transplantation experiments using LSK cells. Validating our previous results, we also found that old mice LSK cells reconstituted the younger mice $(\mathrm{OY})$ as efficiently as can be seen in case of 2-month young donor LSK cells in the control group (YY), although LSKFlk2 ${ }^{+/-}$cells were low in the former group (Fig. 3B). In contrast, young LSKFlk2 ${ }^{+/-}$cells recovered from old recipient mice (YO) were significantly lower than those obtained from other two groups: OY and YY (Fig. 3B). The BM reconstitution efficiency in young recipients with old LSK cells (OY) was significantly higher than "YO" group $\left(58.63 \pm 3.64 \times 10^{6}\right.$ versus $\left.45.0 \pm 2.9 \times 10^{6}, P<0.05\right)$. Overall, this comparative analysis indicated a critical role of $\mathrm{BM}$ microenvironment in hematopoietic reconstitution post transplantation.

It is possible that large number of HSPCs of old donor cells in young recipient mice (OY) were due to better homing and engraftment potential of cells. To examine it, 
A
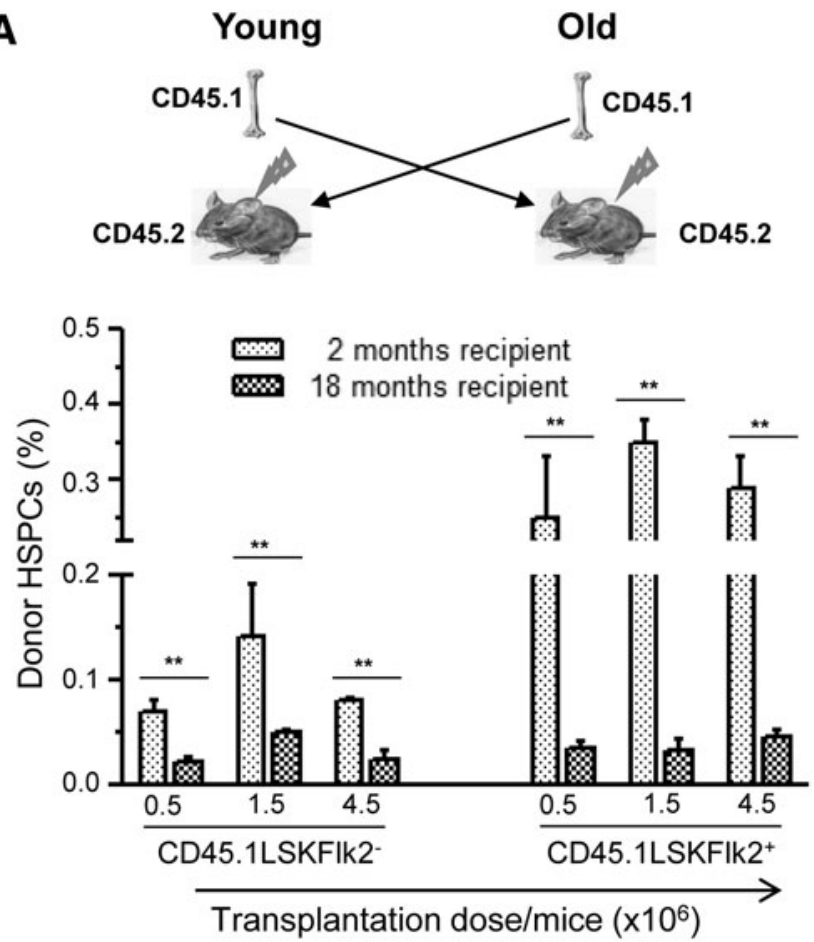

B

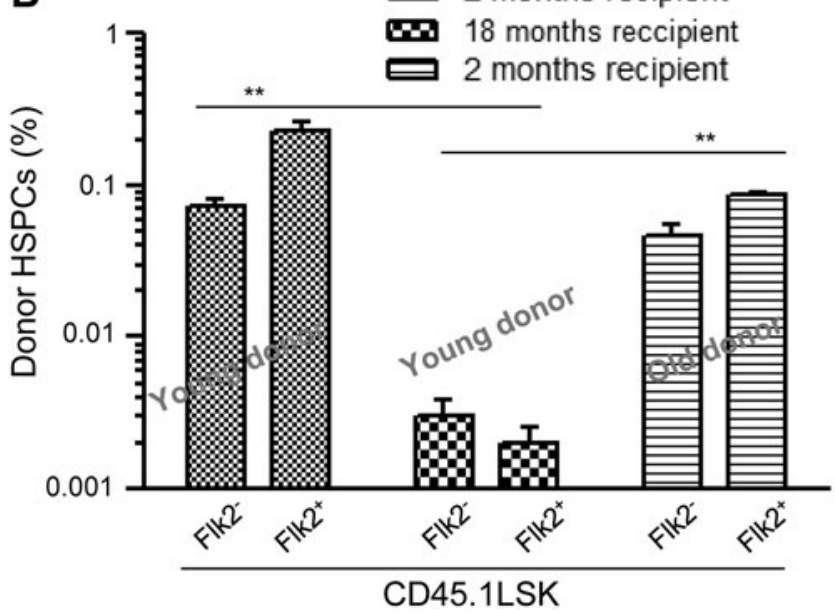

FIG. 3. Reciprocal transplantation of cells. (A) Three different doses $\left(0.5,1.5\right.$ and $4.5 \times 10^{6}$ cells/mice $)$ of BM-MNCs from young and old CD45.1 mice were transplanted in two groups of old and young mice, respectively. After 2 months of transplantation, each group of mice was sacrificed and donor-derived LSKFlk2 ${ }^{+/-}$cells were determined. Number of mice in each group $(n)=6$. $* * P<0.01$. (B) Thirty thousand CD45.1LSK cells of young donor were transplanted in two groups of young and old recipients. The third group of young mice received same number of cells from old mice. After 2 months of transplantation, mice were sacrificed and LSKFlk2 $2^{+-}$cells were determined based on 2 tibias and 2 femurs. Number of mice in each group $(n)=8 . * * P<0.01$.

we performed a short-term competitive marrow engraftment potential assay of young and old cells. Equal proportions of these two cell types were transplanted in 2- and 18-month recipient mice, and the engrafted cells were analyzed after $24 \mathrm{~h}$. As we planned to detect the donor cells (before pro- liferation), a relatively large number of BM-MNCs were transplanted so that few of them can engraft in the marrow. We determined the homing potential of donor cells on the basis of percentage of CD45.1 (Y) and CD45.2GFP $(\mathrm{O})$ cells recovered from sets of femur and tibia from each recipient. The results showed that irrespective of the recipient age, the engraftment potential of young cells was higher than old cells, which was more prominent in case of young donor cells to young recipient (YY) (Fig. 4A, bottom). Although we do not claim that all engrafted cells belonged to HSPCs, this experiment suggested that the young BM cells have better engraftment potential compared to old cells in a competitive environment.

The engraftment of cells on marrow stroma is governed by the expression of cell surface molecules on HSPCs. Flow cytometric analysis for the expression of CD44 and CD29 surface adhesion molecules in LSK cells revealed no significant difference between young and old mice. However, more CXCR $4^{+} \mathrm{LSK}$ cells in young were found compared to old mice and implied its' potential role for above differential engraftments (Fig. 4B).

\section{ROS level and apoptotic death are augmented in stromal cells with the age}

As reciprocal transplantation experiments implied a probable role of stromal cells in hematopoietic reconstitution, we examined the cause and effect on LSK and CD45 ${ }^{-}$cells. ROS are the metabolites that have been held responsible for cellular injury beyond a critical level. ROS are found to accumulate in the mammalian tissues with age. The mitochondrial ROS level was determined by staining BM cells with MitoSOX red dye, which is a superoxide indicator dye that specifically measures ROS content of the organelle. Before staining with the dye, $\mathrm{BM}$ cells were labeled for two distinct compartments, one LSK cells and the other stromal cells (CD45 $)$. The percentage of MitoSOX-stained cells in stromal population at different ages of the mice is shown in the representative dot plots (Fig. 5A). Comparative analysis showed that ROS accumulation steadily increased with age in both populations, but was significantly higher $(P<0.01)$ in stromal compartment compared to LSK $(26.46 \% \pm 6.38 \%$ versus $4.5 \% \pm 1.3 \%)$ of old mice. Above results were validated by microscopic analysis where a time-dependent accumulation of ROS in the stromal cells was observed (Fig. 5B).

To determine apoptotic cells in LSK and CD45- compartments, we stained BM cells for Annexin-V. Flow cytometric analysis revealed that there was a steep increase of apoptotic cells in stromal compartment from 12 months onward (Fig. 5C). A sharp ninefold increase of apoptosis was observed in 12-month-old stromal cells compared to young cells. On the other hand, LSK cells were not much affected. Overall, these results perhaps indicated susceptibility of stromal cells to ROS and concluded that its' subsequent stress may lead to high apoptotic death in older cells.

\section{NAC treatment attenuates ROS-mediated cellular damage in old stromal cells}

Cellular antioxidant defense system plays a crucial role in balancing ROS level below the critical limit. To understand 

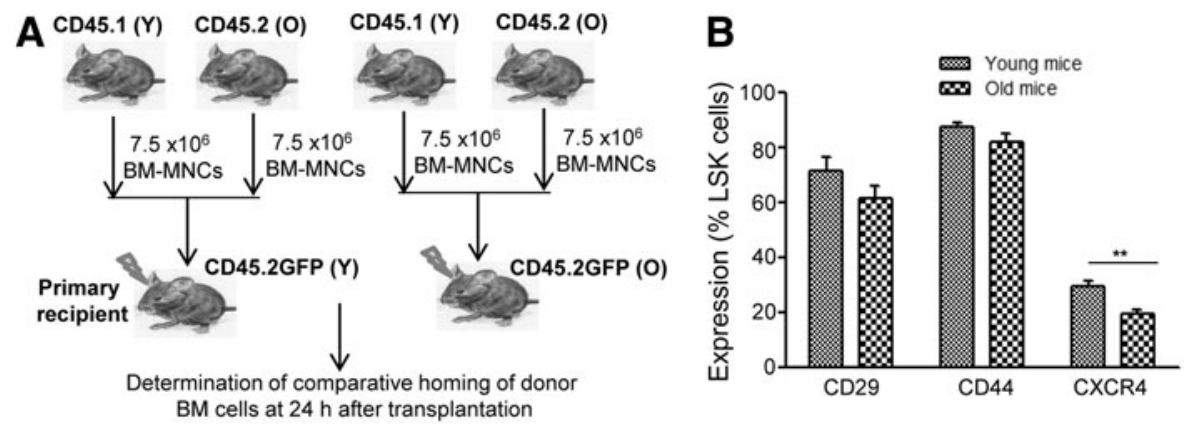

\begin{tabular}{|cccc|}
\hline Recipient & CD45.1\%(Y) & CD45.2 \% (O) Significance \\
\hline CD45.2GFP (Y) & $3.34 \pm 0.17$ & $1.54 \pm 0.15$ & $\mathrm{P}<0.01$ \\
CD45.2GFP (O) & $2.67 \pm 0.38$ & $1.16 \pm 0.32$ & $\mathrm{P}<0.05$ \\
\hline
\end{tabular}

FIG. 4. Competitive homing potential of young $(\mathrm{Y})$ and old $(\mathrm{O})$ donor cells. (A) The scheme of the experiment has been presented at the top. Equal number of young and old donor BM-MSCs were transplanted in young and old recipients. After $24 \mathrm{~h}$ of transplantation, percentage donorderived cells $\left(\mathrm{CD} 45.1^{+} / \mathrm{CD} 45.2^{+}\right.$cells in young and old CD45.2GFP mice) were determined. Number of mice in each group $(n)=5$. (B) Cell adhesion and homing molecules in LSK cells. Flow cytometric analyses of CD44, CD29, and CXCR4 in young and old mice are shown. Number of mice in each group $(n)=3$. $* * P<0.01$. whether an ROS quencher can rejuvenate old stromal cells (18 months) for restoration of physiological functions, we cultured them in the presence of NAC, which is a potent pharmacological compound that quenches ROS. The results clearly demonstrated that ROS significantly declined with concomitant reduction of apoptosis in old stromal cells (Table 1). No such effect was observed in case of young stromal cells. To assess whether reduction of ROS level in old stromal cells can influence adhesion and maintenance of HPSCs, we conducted coculture experiments. LSK cells were cocultured with NAC-treated or -untreated stromal cells of two extreme age groups of mice for $72 \mathrm{~h}$. While no significant difference in the expressions of CD29, CD44, and CXCR4 in LSK background was observed (data not shown) either in old or young mice, there was apparent improvement of LSKFlk2 $2^{-}$cells in NAC-treated old stromal cells (Table 1).

\section{Curcumin treatment reduces ROS load in stromal cells, minimizes stress-responsive gene expressions, and improves BM cellularity}

As ROS were found to be accumulated in perturbed stromal cells, we tested another potent quencher of cellular ROS in vivo, which is curcumin [26], and subsequently determined BM cellularity in older mice. The ROS level has

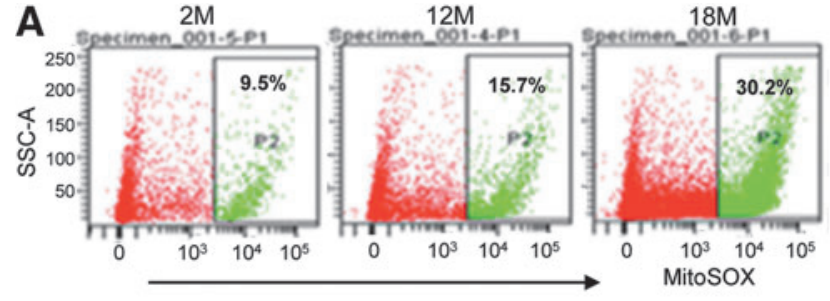

B
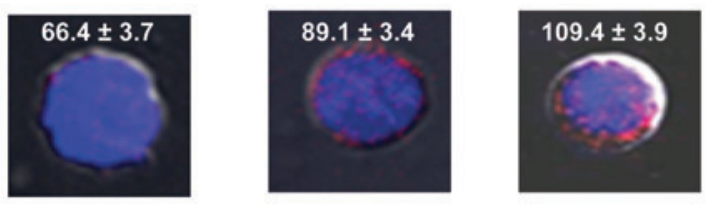

C
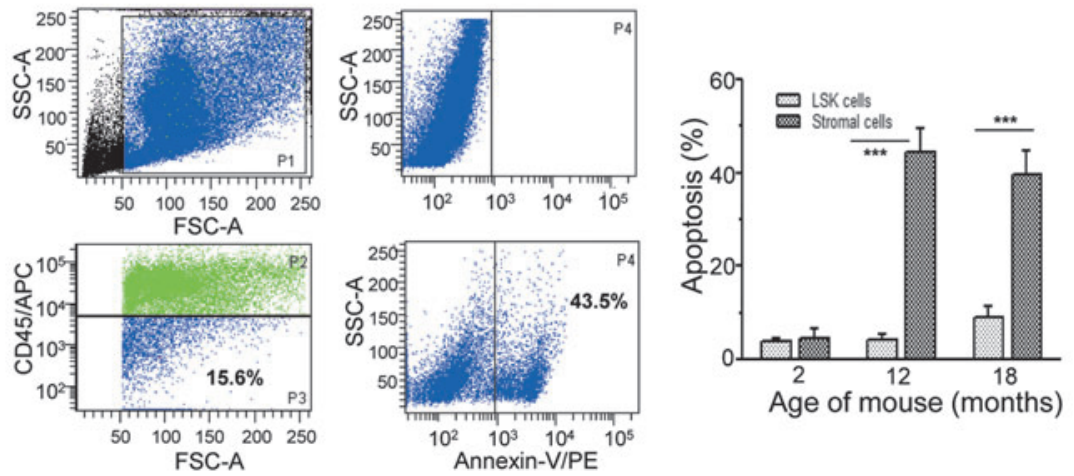

FIG. 5. Accumulation of ROS and cellular apoptosis. (A) Representative dot plots show percentage of stromal $\left(\mathrm{CD} 45^{-}\right)$cells that were stained with MitoSOX dye. The bar diagram shows relative proportion of LSK and stromal cells at different ages that are stained with the dye. Number of mice in each group $(n)=4$. $* * P<0.01$. (B) Confocal images show MitoSOX dye-stained stromal cells at 2,12 , and 18 months of age. Comparative staining of cells in arbitrary scale is also shown. Number of mice in each group $(n)=2$. (C) Representative dot plots show percentage of stromal $\left(\mathrm{CD} 45^{-}\right)$cells stained with Annexin-V. The bar diagram shows relative proportion of LSK and stromal cells at different ages that are stained with Annexin-V. Number of mice in each group $(n)=4$. $* * * P<0.001$. ROS, reactive oxygen species. 
Table 1. Effects on N-Acetyle Cysteine on Reactive Oxygen Species and Apoptosis in Stromal Cells

\begin{tabular}{|c|c|c|c|c|}
\hline Experimental conditions & $\begin{array}{c}\text { Mitox-ROS } \\
(\%)\end{array}$ & $\begin{array}{c}\text { Annexin- } V^{+} \\
\text {cells }(\%)\end{array}$ & $\begin{array}{l}\text { LSKFlk2 } \\
\text { cells }(\%)\end{array}$ & $\begin{array}{l}\text { LSKFlk2- } \\
\text { cells }(\%)\end{array}$ \\
\hline “'O” stromal cells (w/o NAC) & $41.1 \pm 4.96$ & $30.7 \pm 0.45$ & $69.6 \pm 12.2$ & $30.4 \pm 10.2$ \\
\hline “O”' stromal cells (with NAC) & $21.2 \pm 2.29^{\mathrm{a}}$ & $12.4 \pm 5.95^{\mathrm{a}}$ & $45.0 \pm 5.0^{\mathrm{a}}$ & $57.0 \pm 4.29^{\mathrm{a}}$ \\
\hline "Y" stromal cells (w/o NAC) & $21.4 \pm 1.65$ & $24.2 \pm 10.9$ & $41.7 \pm 8.35$ & $58.4 \pm 8.35$ \\
\hline "Y" stromal cells (with NAC) & $14.4 \pm 7.46^{\mathrm{b}}$ & $21.6 \pm 3.4^{\mathrm{b}}$ & $64.5 \pm 7.84^{\mathrm{b}}$ & $35.5 \pm 7.81^{\mathrm{b}}$ \\
\hline \multirow[t]{2}{*}{ Significance } & ${ }^{\mathrm{a}} P<0.03$ & ${ }^{\mathrm{a}} P=0.05$ & ${ }^{\mathrm{a}} P=0.1$ & ${ }^{\mathrm{a}} P=0.05$ \\
\hline & ${ }^{\mathrm{b}} P=0.94$ & ${ }^{\mathrm{b}} P=0.36$ & ${ }^{\mathrm{b}} P=0.15$ & ${ }^{\mathrm{b}} P=0.15$ \\
\hline
\end{tabular}

Number of mouse in each group $=3$.

$\mathrm{a}, \mathrm{b}$ : significance.

NAC, N-acetyle cysteine; ROS, reactive oxygen species.

been linked with the cellular GSH and GSSG status; we therefore analyzed their concentrations in stroma cells of old mice (Group I \& III). It was apparent from the analysis that curcumin treatment in old mice significantly improved GSH level $(7.06 \pm 0.87$ vs. $0.41 \pm 0.14, P<0.01)$ in $\mathrm{CD}^{2} 5^{-}$com- partment of BM cells compared to that of untreated mice (Fig. 6A). On the contrary, no apparent changes in GSSG and total GSH levels were observed between the two groups. Interestingly, curcumin treatment was also associated with decreased expressions of oxidative stress response genes.
FIG. 6. Effect of curcumin treatment on ROS levels in stromal cells and total bone marrow cellularity in old mice. (A) Mice were treated with curcumin as per the scheme; cellular GSSG, GSH, and total-GSH are compared with sham control group. Number of mice in each group $(n)=3$. $* * P<0.01$. (B) Stromal cells of above curcumin-treated mice were subjected to the analysis of oxidative stress-responsive gene expressions. Fold change was calculated with respect to that in young mice. Number of mice in each group $(n)=3$. $* P<0.05$; $* * P<0.01$. (C) Stromal cells of above curcumintreated groups of mice were subjected to analysis of antiapoptotic and hematopoietic growth factor gene expressions. Number of mice in each group $(n)=2-4 . \quad * P<0.05$; $* * P<0.01$. (D) Curcumin-untreated/ treated groups of mice were sacrificed after 15 days of transplantation; total BM-MNCs, stromal cell number, and ROS levels were determined and compared with young mice (untreated). Number of mice in each group $(n)=6$. $* P<0.05$; $* * P<0.01$; $* * * P<0.001$.
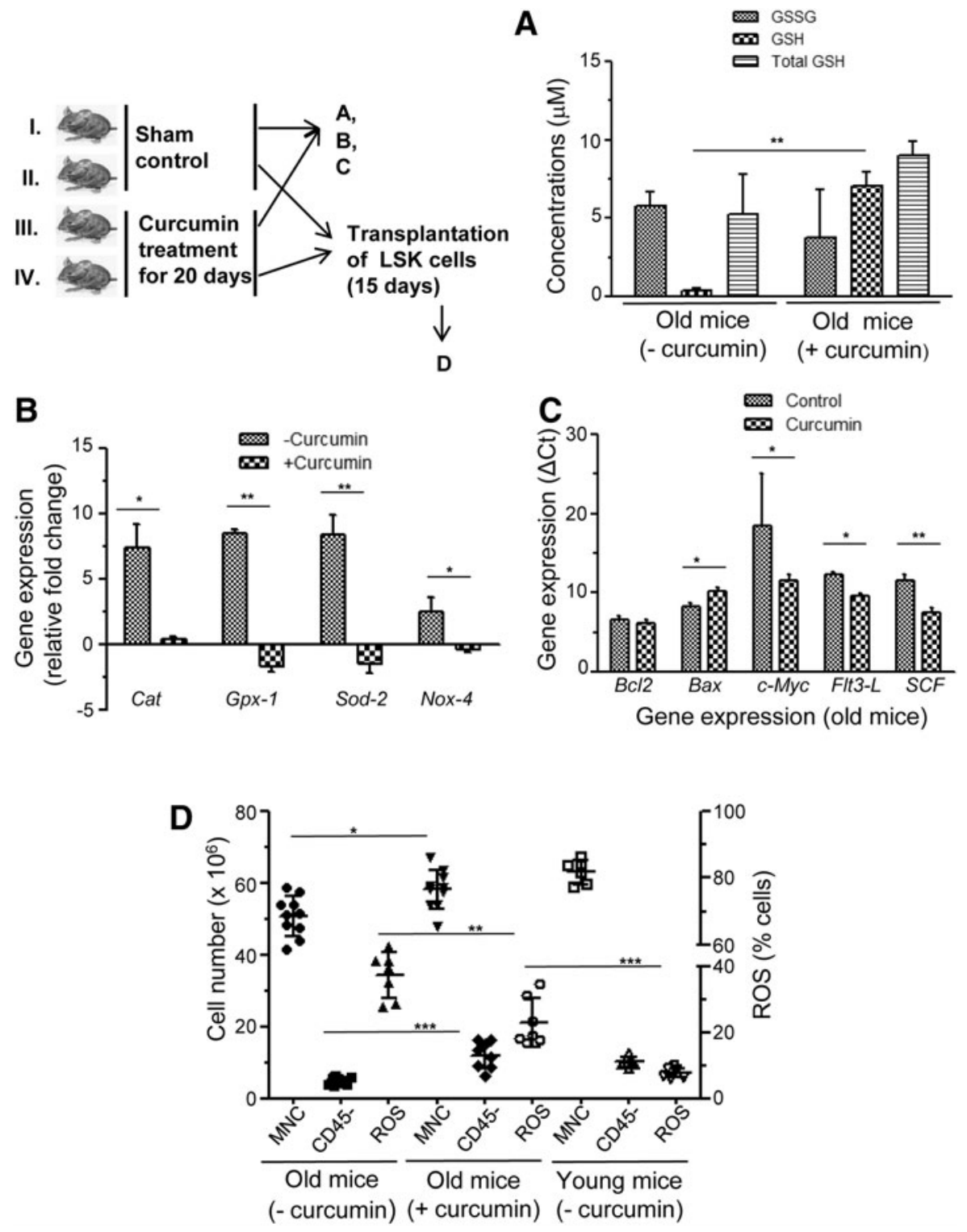
Catalase, superoxide dismutase-2 (Sod-2), glutathione peroxidase (Gpx-1), and NADPH oxidase $4($ Nox-4) levels were significantly upregulated in old mice stromal cells as evidenced by fold change of gene expressions relative to young control stromal cells (Fig. 6B). All these stress-responsive gene expressions significantly declined in old stromal cells when treated with curcumin.

Subsequent analysis showed that $\mathrm{Bcl}-2$ gene expression did not change much between the samples, whereas Bax was significantly suppressed in curcumin-treated stromal cells (Fig. 6C). On the other hand, $c-M y c$ expression was increased after curcumin treatment. Furthermore, early acting cytokines $F l t-3 L$ and $S C F$ gene expression was also found to improve after curcumin treatment to the stromal cells compared to untreated control (Fig. 6C). Correlating GSH/ GSSG results with gene expression analyses, we concluded that curcumin treatment improves cellular antioxidant defense system, triggers antiapoptotic signals, and enhances the expression of hematopoietic growth factors.

Later, group II (curcumin untreated) and IV (curcumin treated) mice received LSK cells following sublethal irradiation (scheme in Fig. 6). After 15 days of transplantation, mice were sacrificed for analysis of ROS in stromal compartment, including BM, and stromal cellularity. Interestingly, curcumin treatment resulted in significant improvement of total BM-MNCs $(58.34 \pm 1.7$ vs. $51.0 \pm 1.8$, $P<0.05)$ and $\mathrm{CD} 45^{-}$cells $(12.24 \pm 1.35$ vs. $5.0 \pm 0.32$, $P<0.01)$ within 15 days of transplantation compared to control mice, which were not treated (Fig. 6D). The BM cellularity of these treated mice was comparable to control mice (2 months), however, ROS levels in stromal cells were still higher than those of young mice (Fig. 6D). Overall, these results indicated the role of ROS, as its' reduction resulted in proliferation of old stromal cells and improvement of BM cellularity.

\section{Discussion}

Studies on aging of HSCs have been pursued mainly in two perspectives: (a) increase of myeloid lineage biased with age and (b) low hematopoietic reconstitution in aging recipients. Aging of hematopoietic system has been found responsible for the development of immune dysfunction (e.g., lymphopenia) and BM failure syndromes [27-31]. The reasons for stem cells aging are unraveling, however, collective evidences suggest that lineage potential, homing efficiency, change of transcriptional activity, genomic integrity, and cellular metabolism in aging HSCs are intrinsically controlled $[17,32]$. Yet, the complex interaction of HSCs with microenvironment (extrinsic influence) in aging $\mathrm{BM}$ remains elusive. In recent past, it has been revealed that microenvironment plays a critical role in protecting HSCs from cytotoxic agents and metabolic by-products, and in maintaining dormancy, etc. [33,34]. These suggest that understanding BM microenvironment in pathological condition (especially in aged mouse stromal cells) and its' potential reversal holds the key for development of innovative therapeutics. In this study, we have shown that hematopoietic reconstitution was compromised in aging BM due to perturbation of stromal cells, a vital component of stem cell niche, which, however, was partly reversed by reducing the effect of causative agent.
Based on the analysis of HSPC pool in young and old mice, we could not find a significant difference, although earlier studies showed that HSCs get accumulated with age [4]. The variation of these results is probably attributed to the phenotype restrictions; we followed LSKFlk2 $2^{-/+}$for analysis, whereas earlier studies were based on LSKCD34 ${ }^{-/ \mathrm{lo}}$ [4] or LSKFlk2 ${ }^{-} \mathrm{CD} 150^{+} \mathrm{CD} 48^{-}$phenotype [35]. Despite no significant differences of LSKFlk $2^{-1+}$ cells in old and young mice being observed, we could ably demonstrate that GEMM and GM colonies got significantly increased in the former group. This further strengthens the view of increasing myeloid-specific HSC subset in aging mice $[4,15]$. The serial transplantation results showed gradual progress of HSPCs toward replicative senescence [13]; although it was more pronounced in older mice. This may be attributed to the impaired homing and engraftment potential of the cells.

Short-term homing experiment suggested that young cells were more potent in homing and engraftment, which was further supported by relatively high expression of CXCR4 in LSK cells compared to that in old cells [36]. However, these results did not justify the outcome of reciprocal transplantation experiments as the donor cells of 18-month-old mice (OY) performed better than young mice (YO) in terms of LSKFlk2 ${ }^{-/+}$cell number and marrow regeneration potential. These data indicated BM microenvironment as one of the probable factors that could influence the transplantation outcome.

There has been increasing evidence in mammals that intracellular accumulation of ROS with age renders oxidative stress leading to damage/dysregulation of tissues, specifically BM [30]. At an elevated ROS level, impaired DNA repair mechanism was found to contribute in BM failure in case of Fanconi anemia [37]. Besides BM, retinal pigmental epithelium [38], cardiomyocyte [39], and renal tubular epithelium [40] were shown affected due to physiological or pathophysiological ROS stress. Furthermore, there were increasing evidences linking ROS at high levels damaging different cellular macromolecules. To protect themselves, cells develop numerous intricate antioxidant defense systems, such as enzymatic scavengers, nonenzymatic glutathione, and vitamins [41]. ROS was shown to have a negative role in marrow regeneration, where ectopic overexpression of ROS-detoxifying enzymes improved HSC regeneration capacity in mice [42]. This study correlated mitochondrial ROS level of stromal cells with cellular apoptosis; thus, we drew a general conclusion that BM niche got deteriorated with age. This result has been supported by an earlier report on accelerated senescence of human BMderived MSCs in aging subjects [10].

Interestingly, ROS level in the HSPC compartment and subsequent apoptosis with age was much lower, which motivated us to propose that apoptosis in stromal compartment was ROS dependent. Low ROS level in HSPCs was possibly due to two reasons: (a) metabolically less active cells and/or dormant cells consume less $\mathrm{O}_{2}$ and (b) presence of robust ROS-detoxifying system. Furthermore, a recent study has shown that connexin-43, expressed in HSCs, exerts a protective role by regulating intracellular ROS content through the transfer to hematopoietic microenvironment [34]. On the other hand, high proliferative nature of stromal cells consumes more $\mathrm{O}_{2}$ and hence increases the ROS level and is self-exposed to oxidative stress. This was probably 
due to the presence of low antioxidant levels in BM stromal cells [43]. GSH is the hallmark redox buffer in all living cellular systems, so in this study, we hypothesize that the balance between GSH and GSSG was disturbed in the aging mice stromal cells at low GSH level. Taken together, these results explain why we observed inhibitory effect of ROS on stromal cells and not in HSPCs.

It has been shown that naturally occurring antioxidant agent, like curcumin can ameliorate age-related changes that are associated with oxidative damage by protecting lipid peroxidation, protein carbonylation, and mitochondrial permeability transition $[44,45]$. Our study clearly indicated the role of curcumin in the reduction of oxidative stress in BM stormal cells, as GSH and ROS levels were inversely modulated and expressions of stress-responsive genes Nox-1, Sod-2, and Gpx-1 declined. How curcumin suppressed the ROS level was not studied in this investigation, yet we believe that downregulation of commonly stress-activated signalling pathways leading to the activation of NF- $\kappa \mathrm{B}$, p38 MAPK, and JNK could be the possible reasons $[46,47]$

In this study, we evidenced overall improvement of curcumin-treated old stromal cells compared to age-matched control mice by reduction of ROS levels. The fact that cucumin reduces oxidative stress and apoptosis and improves proliferation has been documented in the case of aging retinal pigmental epithelium [38]. While curcumin induces apoptosis in many tumor cells, in contrast to the proapoptotic role, its' antiapoptotic function was found to be beneficial in BM stromal cells. This antiapoptotic function of curcumin has also been demonstrated by inhibition of ROS generation and the blockage of JNK function in the case of human breast cancer cells [48]. High expression of $c-M y c$ and low expression of Bax in curcumin-treated stromal cells suggested that, in part, apoptotic stimuli of ROS were suppressed in favor of proliferation [49]. In addition, high expression of early acting cytokines Flt3-ligand and $S C F$ genes [50] supported the notion that hematopoietic reconstituting ability of older mice was rejuvenated by treatment with curcumin. Although we observed some benefit of using NAC-treated old stromal cells in culture, it was insignificant to what was reported earlier in which NAC-treated NOD-SCID mice showed high engraftment and multilineage differentiation of human HSCs [51].

Overall, this study proposes that ROS accumulation in aging BM stromal cells leads to perturbation of stromal functions. The treatment of antioxidants like curcumin can partly overcome one of the problems associated with aging that is low marrow reconstitution efficiency. This study did not look into the myeloid-biased hematopoietic imbalance in aging mice after curcumin treatment, which needs to be addressed separately. Given that stem cell population in $\mathrm{BM}$ does not change much with the age, why is BM reconstitution significantly compromised? We anticipate that chronic stress adversely contributes to stromal cells in aging mice, which are irreversibly degenerated during conditioning (e.g., radiation), thereby unable to extend support to hematopoietic compartment unless treated with antioxidant [52]. This preliminary study proposes that preconditioning of $\mathrm{BM}$ with antioxidants like curcumin would be necessary before hematopoietic reconstitution in older recipients.

\section{Acknowledgment}

A.M. is thankful to Department of Science and Technology, Government of India for partial funding to this study.

\section{Author Disclosure Statement}

No competing financial interest exists.

\section{References}

1. Anthony BA and DC Link. (2014). Regulation of hematopoietic stem cells by bone marrow stromal cells. Trends Immunol 35:32-37.

2. Morrison SJ, AM Wandycz, K Akashi, A Globerson and IL Weissman. (1996). The aging of hematopoietic stem cells. Nat Med 2:1011-1016.

3. Liang Y, G Van Zant and SJ Szilvassy. (2005). Effects of aging on the homing and engraftment of murine hematopoietic stem and progenitor cells. Blood 106:1479-1487.

4. Sudo K, H Ema, Y Morita and H Nakauchi. (2000). Ageassociated characteristics of murine hematopoietic stem cells. J Exp Med 192:1273-1280.

5. Kim M, HB Moon and GJ Spangrude. (2003). Major agerelated changes of mouse hematopoietic stem/progenitor cells. Ann N Y Acad Sci 996:195-208.

6. Kamminga LM, R van Os, A Ausema, EJ Noach, E Weersing and B Dontje. (2005). Impaired hematopoietic stem cell functioning after serial transplantation and during normal aging. Stem Cells 23:82-92.

7. Nijnik A, L Woodbine, C Marchetti, S Dawson, T Lambe, C Liu, NP Rodrigues, TL Crockford, E Cabuy, et al. (2007). DNA repair is limiting for haematopoietic stem cells during ageing. Nature 447:686-690.

8. Rossi DJ, D Bryder, J Seita, A Nussenzweig, J Hoeijmakers and IL Weissman. (2007). Deficiencies in DNA damage repair limit the function of haematopoietic stem cells with age. Nature 447:725-729.

9. Beausejour C. (2007). Bone marrow-derived cells: The influence of aging and cellular senescence. Handb Exp Pharmacol 180:67-88.

10. Stenderup K, J Justesen, C Clausen and M Kassem. (2003). Ageing is associated with decreased maximal life span and accelerated senescence of bone marrow stromal cells. Bone 33:919-926.

11. van Deursen JM. (2014). The role of senescent cells in ageing. Nat Rev 509:439-446.

12. Ogden DA and HS Mickliem. (1976). The fate of serially transplanted bone marrow cell populations from young and old donors. Transplantation 22:287-293.

13. Harrison DE and CM Astle. (1982). Loss of stem cell repopulating ability upon transplantation. Effects of donor age, cell number and transplantation procedure. J Exp Med 156:1767-1779.

14. Muller-Sieburg CE and HB Sieburg. (2006).Clonal diversity of the stem cell compartment. Curr Opin Hematol 13: 243-248.

15. Muller-Sieburg CE, RH Cho, M Thoman, B Adkins and HB Sieburg. (2002). Deterministic regulation of hematopoietic stem cell self-renewal and differentiation. Blood 100:1302-1309.

16. Chambers SM, CA Shaw, C Gatza, CJ Fisk, LA Donehower and MA Goodell. (2007). Aging hematopoietic stem cells decline in function and exhibit epigenetic dysregulation. PLoS Biol 5:e201. 
17. Chen C, Y Liu, Y Liu and P Zheng. (2009). mTOR regulation and therapeutic rejuvenation of aging hematopoietic stem cells. Sci Signal 2:ra75.

18. Ito K, A Hirao, F Arai, K Takubo, S Matsuoka, K Miyamoto, M Ohmura, K Naka, K Hosokawa, Y Ikeda and T Suda. (2006). Reactive oxygen species act through p38 MAPK to limit the lifespan of hematopoietic stem cells. Nat Med 12: 446-451.

19. Morrison S and AC Spradling. (2008). Stem cells and niches: mechanisms that promote stem cell maintenance throughout life. Cell 132:598-611.

20. Chatterjee S, RK Dutta, P Basak, P Das, M Das, JA Pereira, M Chaklader, S Chaudhuri and S Law. (2010). Alteration in marrow stromal microenvironment and apoptosis mechanisms involved in aplastic anemia: An animal model to study the possible disease pathology. Stem Cells Inter 2010: ID 932354.

21. Marsh JCW, J Chang, NG Testa, JM Hows and TM Dexter. (1991). In vitro assessment of marrow 'stem cell' and stromal cell function in aplastic anaemia. Br J Haematol 78:258-267.

22. Andre T, N Meuleman, B Stamatopoulos, BC De, K Pieters, D Bron and L Lagneaux. (2013). Evidences of early senescence in multiple myeloma bone marrow mesenchymal stromal cells. PLoS One 8:e59756.

23. Vas V, C Wandhoff, K Dorr, A Niebel and H Geiger. (2012). Contribution of an aged microenvironment to agingassociated myeloproliferative disease. PLoS One 7:e31523.

24. Roy S, S Javed, SK Jain, SS Majumdar and A Mukhopadhyay. (2012). Donor hematopoietic stem cells confer long-term marrow reconstitution by self-renewal divisions exceeding to that of host cells. Plos One 7:e50693.

25. Adolfsson J, OJ Borge, D Bryder, K Theilgaard-Monch, I Astrand-Grundstrom, E Sitnicka, Y Sasaki and SE Jacobsen. (2001). Up-regulation of Flt3 expression within the bone marrow $\mathrm{Lin}^{-} \mathrm{Sca}^{+} \mathrm{c}-\mathrm{kit}^{+}$stem cell compartment is accompanied by loss of self-renewal capacity. Immunity 15:659-669.

26. Biswas SK, D McClure, LA Jimenez, IL Megson and I Rahman. (2005). Curcumin induces glutathione biosynthesis and inhibits NF- $\mathrm{KB}$ activation and interleukin 8 release in alveolar epithelial cells: Mechanism of free radical scavenging activity. Antioxid Redox Signal 7:32-41.

27. Rothstein G. (1993). Hematopoiesis in the aged: a model of hematopoietic dysregulation? Blood 82:2601-2604.

28. Lichtman MA and WJ Williams. (2001). Haematology in the Aged. 6th ed. New York, McGraw Hill Books.

29. Richardson C, S Yan and CG Vestal. (2015). Oxidative stress, bone marrow failure, and genome instability in hematopoietic stem cells. Int J Mol Sci 16:2366-2385.

30. Tummala H, M Kirwan, AJ Walne, U Hossain, N Jackson, C Pondarre, V Plagnol, T Vulliamy and I Dokal. (2014). ERCC6L2 mutations link a distinct bone-marrow-failure syndrome to DNA repair and mitochondrial function. Am J Hum Genet 94:246-256.

31. Eliades A, S Matsuura and K Ravid. (2012). Oxidases and reactive oxygen species during hematopoiesis: a focus on megakaryocytes. J Cell Physiol 227:3355-3362.

32. Rossi DJ, D Bryder, JM Zahn, H Ahlenius, R Sonu, AJ Wagers and IL Weissman. (2005). Cell intrinsic alterations underlie hematopoietic stem cell aging. Proc Natl Acad Sci USA 102:9194-9199.

33. Wilson A and A Trumpp. (2006). Bone-marrow haematopoietic stem cell niches. Nat Rev Immunol 6:93-106.
34. Ishikawaa ET, D Gonzalez-Nietob, G Ghiaurb, SK Dunna, AM Fickerb, B Muralib, M Madhub, DE Gutsteinc, GI Fishmanc, CC Barrioe and JA Cancelasa. (2012). Connexin-43 prevents hematopoietic stem cell senescence through transfer of reactive oxygen species to bone marrow stromal cells. Proc Natl Acad Sci USA 109: 9071-9076.

35. Oguro H, L Ding and SJ Morrison. (2013). SLAM family markers resolve functionally distinct subpopulations of hematopoietic stem cells and multipotent progenitors. Cell Stem Cell 13:102-116.

36. Peled A, I Petit, O Kollet, M Magid, T Ponomaryov, T Byk, A Nagler, H Ben-Hur, A Many, et al. (1999). Dependence of human stem cell engraftment and repopulation of NOD/SCID mice on CXCR4. Science 283: 845-848.

37. Zhang X, J Li, DP Sejas and Q Pang. (2005). Hypoxiareoxygenation induces premature senescence in FA bone marrow hematopoietic cells. Blood 106:75-85.

38. Zhu W, Y Wu, YF Meng, JY Wang, M Xu, JJ Tao and J Lu. (2015). Effect of curcumin on aging retinal pigment epithelial cells. Drug Des Devel Ther 9:5337-5344.

39. von Harsdorf R, PF Li and R Dietz. (1999). Signaling pathways in reactive oxygen species-induced cardiomyocyte apoptosis. Circulation 99:2934-2941.

40. Ding W, L Yang, M Zhang and Y Gu. (2012). Reactive oxygen species-mediated endoplasmic reticulum stress contributes to aldosterone-induced apoptosis in tubular epithelial cells. Biochem Biophy Res Commun 418:451456.

41. Poljsak B, D Šuput and I Milisav. (2013). Achieving the balance between ROS and antioxidants: when to use the synthetic antioxidants. Oxid Med Cell Long 2013:Article ID 956792.

42. Miao W, R XuFeng, MR Park, H Gu, L Hu, JW Kang, S Ma, PH Liang, Y Li, et al. (2013). Hematopoietic stem cell regeneration enhanced by ectopic expression of ROSdetoxifying enzymes in transplant mice. Mol Ther 21:423432.

43. Ebert R, M Ulmer, S Zeck, J Meissner-Weigl, D Schneider, H Stopper, N Schupp, M Kassem and F Jakob (2006). Selenium supplementation restores the antioxidative capacity and prevents cell damage in bone marrow stromal cells in vitro. Stem Cells 24:1226-1235.

44. Trujillo J, YI Chirino, E Molina-Jijón, AC AndéricaRomero, E Tapia and J Pedraza-Chaverrí. (2013). Renoprotective effect of the antioxidant curcumin: Recent findings. Red Biol 1:448-456.

45. Jat D, P Parihar, SC Kothari and MS Parihar. (2013). Curcumin reduces oxidative damage by increasing reduced glutathione and preventing membrane permeability transition in isolated brain mitochondria. Cell Mol Biol 59(Suppl):OL1899-OL1905.

46. Moon-Sook W, J Soo-Hyun, K So-Young, H Jin-Won, K Kwang-Ho, K Won-Ki and K Hee-Sun. (2005). Curcumin suppresses phorbol ester-induced matrix metalloproteinase9 expression by inhibiting the PKC to MAPK signaling pathways in human astroglioma cells. Biochem Biophy Res Commu 335:1017-1025.

47. Evans JL, ID Goldfine, BA Maddux and GM Grodsky. (2002). Oxidative stress and stress-activated signalling pathways: a unifying hypothesis of type 2 diabetes. Endocrine Rev 23:599-622. 
48. Somasundaram S, NA Edmund, DT Moore, GW Small, YY Shi and RZ Orlowski. (2002). Dietary curcumin inhibits chemotherapy-induced apoptosis in models of human breast cancer. Cancer Res 62:3868-3875.

49. Wilson A, MJ Murphy, T Oskarsson, K Kaloulis, MD Bettess, GM Oser, AC Pasche, C Knabenhans, HR Macdonald and Trumpp. (2004). c-Myc controls the balance between hematopoietic stem cell self-renewal and differentiation. Genes Dev 18:2747-2763.

50. Lyman SD, K Brasel, AM Rousseau and DE Williams. (1994). The flt3 ligand: a hematopoietic stem cell factor whose activities are distinct from steel factor. Stem Cells 12 (Suppl 1):99-107.

51. Cheng H, Y Gao, M Shi, Y Liu, Z Hu, J Xu, L Qiu, W Yuan, AY Leung, YG Yang and T Cheng. (2014). The antioxidant $\mathrm{N}$-acetyl-L-cysteine increases engraftment of human hematopoietic stem cells in immune-deficient mice. Blood 124:e45-e48.
52. Shen $\mathrm{H}, \mathrm{H}$ Yu, PH Liang, $\mathrm{H}$ Cheng, $\mathrm{R}$ Xufeng, $\mathrm{Y}$ Yuan, $\mathrm{P}$ Zhang, CA Smith and T Cheng. (2012). An acute negative bystander effect of $\gamma$-irradiated recipients on transplanted hematopoietic stem cells. Blood 119:3629-3637.

Address correspondence to: Asok Mukhopadhyay, PhD Stem Cell Biology Laboratory National Institute of Immunology Aruna Asaf Ali Marg New Delhi-110067 India

E-mail: ashok@nii.ac.in

Received for publication December 21, 2015

Accepted after revision April 30, 2016

Prepublished on Liebert Instant Online May 2, 2016 